\title{
An Image Reconstruction Algorithm based on Sparse Representation for Image Compressed Sensing
}

\author{
Shuyao Tian ${ }^{1}$, Liancheng Zhang ${ }^{1}$ and Yajun Liu ${ }^{2}$ \\ ${ }^{1}$ College of Electronics and Control Engineering, North China Institute of Aerospace Engineering, \\ Langfang, China \\ ${ }^{2}$ College of Mechanical and Electrical Engineering, Hebei Normal University of Science \& \\ Technology, Qinhuangdao, China
}

Received: November 22, 2020. Revised: May 10, 2021. Accepted: May 28, 2021. Published: June 4, 2021.

\begin{abstract}
It is difficult to control the balance between artifact suppression and detail preservation. In addition, the information contained in the reconstructed image is limited. For achieving the purpose of less lost information and lower computational complexity in the sampling process, this paper proposed a novel algorithm to realize the image reconstruction using sparse representation. Firstly, the principle of algorithm for sparse representation is introduced, and then the current commonly used reconstruction algorithms are described in detail. Finally, the algorithm can still process the image when the sparsity is unknown by introducing the sparsity theory and dynamically changing the step size to approximate the sparsity. The results explain that the improved algorithm can not only reconstruct the image with unknown sparsity, but also has advantages over other algorithms in reconstruction time. In addition, compared with other algorithms, the reconstruction time of the improved algorithm is the shortest under the same sampling rate.
\end{abstract}

Keywords-Image reconstruction, Sparse representation, Compressed sensing, PSNR, Reconstruction time.

\section{INTRODUCTION}

$\mathrm{W}$ th the development of machine learning, the idea is introduced into the field of image processing. Learning based image SRR algorithm came into being, and soon became a hot research direction [1-2]. The premise of this algorithm is that the correspondence between HR and LR images can be obtained by learning, and the corresponding mapping relationship can be mastered by training HR and LR image blocks. For the input image to be reconstructed, the nearest data is found in the training data according to some rules corresponding to the corresponding high-resolution training data [3], so as to realize the image SRR. Therefore, in further procession of reconstructing image, we should not only reduce the artificial effect, but also preserve or even restore the details of the image as much as possible in the compressed sensing reconstructed image.

The learning based algorithm was proposed by Onodera et al [4]. This is a SRR method using image sample blocks as training data. The mapping relationship of this method is based on the Markov random field (MRF), and the Markov network is established through the calculation of correlation probability and image spatial relationship [5]. According to the prior probability obtained in the training process, the most suitable position of the LR image to be reconstructed in the Markov network is found, and then the reconstructed HR image is obtained. The algorithm can recover most of the high-frequency information of the image, but at the same time, the accuracy of the Markov network is very high, that is, the data set involved in the training is strict, and the amount of data is large, so the algorithm has low efficiency and poor anti-interference ability. The image reconstruction algorithm based on manifold learning was proposed by Narita et al [6]. This method assumes that there is geometric structure consistency in the manifold space between HR and LR sample image blocks. The local geometric structure of the LR image block to be reconstructed in the training sample is obtained, and its local geometric structure is mapped to the geometric structure space of the HR image training block, and then the HR image is obtained. Allemang et al. introduced the local linear embedding method for the first time, and proposed an image SRR algorithm based on neighborhood embedding [7]. Dhont et al. introduced the principal component analysis method to complete the mapping of the image to its corresponding training sample feature subspace [8]. However, the algorithm is also affected by the high requirement of training set.

With the introduction of sparse representation and compressed sensing theory in the field of digital image processing, Zhao et al. proposed SCSR algorithm [9]. The algorithm assumes that the HR and LR images have similar structure. The existing HR and LR image datasets are used to establish complete dictionaries respectively. The LR image to be reconstructed is represented by the dictionary atoms obtained by training, and the image SRR is realized by using 
the coefficients obtained and the over complete dictionary of HR. This algorithm can fully retain the prior knowledge and reduce the dimension in the training process, which improves the operation efficiency. Yang et al. proposed a new idea [10]. Firstly, the corresponding dictionary was trained by LR image training set. Then, according to the image degradation process, the LR dictionary was used as the input to reverse recover an HR dictionary. The advantage of the algorithm is that it shortens the dictionary training time and improves the implementation efficiency. Zheng et al. developed a new dictionary training method [11], which batch processed the training set to prevent repeated training of data, shorten the dictionary training time and improve the implementation speed. The algorithm proposed by Wang et al. has a variety of sparse constraints [12], and uses its inherent prior knowledge to select a suitable sparse representation model for images in different situations. $\mathrm{Li}$ improved the original dictionary learning algorithm and proposed to establish a joint training dictionary [13], which greatly improved the speed of dictionary training.

However, there is a certain contradiction between the inhibition of artificial effect and the retention of details and it is difficult to control the balance between the inhibition of artificial effect and the retention of details. In addition, the detailed information contained in the reconstructed image is limited. The information lost in the sampling process and computational complexity is low. In order to deal with these problems, this paper proposed a novel algorithm to realize the image reconstruction using sparse representation.

\section{SPARSE REPRESENTATION THEORY}

Sparse representation originated from compressed sensing theory [14], which is an important part. The premise of bioelectrical signal reconstruction in compressed sensing is that bioelectrical signal can be sparsely represented. The principle of compressed sensing is that when the bioelectrical signal can be sparsely expressed, the bioelectrical signal can be projected, and the original bioelectrical signal can be completely restored through the observation matrix [15]. In the field of image processing, the idea of sparse coding is that: natural images generally have similar structure, and all natural images contain a lot of redundant information, so each image can be linearly represented by one or more atoms in an over complete atomic library. For all the atoms in the atomic library, most of the atoms do not need to have zero coefficients. Therefore, the image is sparsely represented by the atomic library. Many researchers and scholars are committed to the in-depth study of sparse representation theory, focusing on finding a more sparse representation and more efficient transformation, and applying the theory to other fields has made great breakthroughs. The idea of sparse representation is proposed to be applied to the recovery and decomposition of bioelectrical signals. Due to the limitation of sparsity in the process of transformation, we must abandon the original linear model and use a more flexible nonlinear model. Sparse representation is widely used in bioelectrical signal processing, which can be used for bioelectrical signal representation and compression. Research shows that sparse representation is a very effective method for bioelectrical signal processing[16]. A large number of results indicate that sparse representation has good effect in the above applications.

For a given image set $D=\left[d_{1}, d_{2}, d_{3}, \ldots, d_{n}\right], D \in R^{m \times n}, m<n$, the training set contains $n$ training images. Among them, each column $d_{i} \in R^{m \times 1}$ represents a training image, and the dimension of each training image is $m \times 1$ dimensional. Assuming that a test image is $y \in R^{m \times 1}$, the training image set can be linearly expressed as

$$
y \approx D x=x_{1} d_{1}+x_{2} d_{2} \ldots+x_{n} d_{n}
$$

Where $x=\left[x_{1} \ldots x_{n}\right] \in R^{n \times 1}$ is the coefficient of the test image expressed linearly in the training image set, and it can also be understood as the projection of the test image. However, in practice, the test image is often occluded or contaminated by noise.

$$
y=D x+\varepsilon
$$

Among them, $\varepsilon \in R^{m \times 1}$ is the error vector, which can be used to represent the occlusion or noise pollution in the image. When the training image set can not accurately represent the measurement image, the error vector can compensate it. $D=\left[d_{1}, d_{2}, d_{3}, \ldots, d_{n}\right], D \in R^{m \times n}, m<n$, is a training image set, and $n$ is much larger than $m$. Therefore, when the test image set $y$ and training image set $D$ are known, it is a NP hard problem to solve the sum of coefficient vectors, and the equation is an underdetermined equation. The underdetermined equation has innumerable solutions. In order to find the most sparse solution, we sparsely limit the coefficient $x$ so that the equation can be transformed into the solution of norm. The sparse representation model can also be represented graphically.

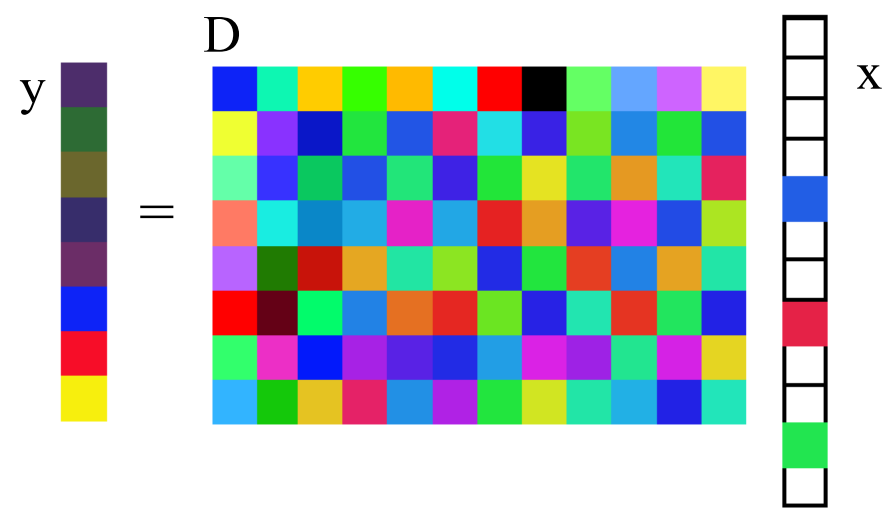

Figure 1. The sparse representation model

In Figure 1, each small square represents an element, and the small squares with different colors represent different values. When the color of the small square is white, it means that the corresponding element value is zero. The more white elements in the figure, the more non-zero items, indicating that $x$ is sparse. The test image $y$ is sparse represented by the training 
image set $D$ and the sparse coefficient $x$.

\section{IMAGE RECONSTRUCTION ALGORITHM BASED ON SPARSE REPRESENTATION}

\section{A. Principle of Image Reconstruction}

The traditional signal reconstruction method is mainly divided into compression stage and reconstruction stage [17]. The signal is sampled in the coding section, and then the sampled value is transformed and compressed to get the important data which can reflect the signal itself. Finally, the encoded value is stored. In the decoding section, the received data is decompressed, and the inverse transform corresponding to the compression stage is performed to obtain the recovery signal.

In the process of sampling, the rate of sampling signal needs to meet the sampling law, that is, the sampling rate should not be less than twice of the signal bandwidth, which has high requirements for the performance of sampling equipment, and the sampling process and compression process are carried out separately, which is a serious waste of time and energy. In view of this situation, compressed sensing theory came into being. The reconstruction algorithm solves two problems in signal reconstruction algorithm.

\section{B. Several Common Image Reconstruction Algorithms}

(1) Basis Pursuit.

Base tracking algorithm is a typical convex optimization algorithm. Its core idea is to reconstruct the original signal with the least base. Base tracking algorithm transforms the difficult minimum norm $\mathrm{L}_{0}$ into the minimum norm $\mathrm{L}_{1}$ under certain conditions, and then transforms a nonlinear programming problem into a linear programming problem.

When the sparse matrix and sensing matrix satisfy the incoherence condition, solving a simpler $\mathrm{L}_{1}$ norm optimization problem will produce a solution equal to $\mathrm{L}_{0}$ norm, so the reconstruction problem is transformed into solving the minimum L1 norm problem under constraint conditions.

$$
\text { Min }\|x\|_{1} \text { s.t. } y=D x
$$

Base tracking algorithm can reconstruct one-dimensional signal well and ensure the sparsity performance, but it has high space-time complexity in the face of large scale.

(2) Matching Tracking Algorithm.

Matching tracking algorithm is one of greedy tracking algorithms, and it is also the basis of various improved algorithms. This algorithm is easy to be studied and implemented. The idea of matching tracking algorithm is simple. In each iteration, we find the atom that best matches the signal residuals from the over complete dictionary, sparse approach the signal, and calculate the signal reconstruction residuals. Then we continue to select the atom that best matches the signal residuals from the over complete dictionary until the signal residuals are small enough or zero, so that the signal goes through a certain number of iterations.

The iteration of MP algorithm mainly includes two steps.

STEP 1. In the process of atom selection, the aim is to find an atom which is most coherent with the current signal residual from the over complete dictionary.

STEP 2. Residual update. Update residuals is to compare the current signal residuals with the original signal residuals after selecting atoms.

The linear combination subtraction is used as the new residual and the signal residual of the next iteration. Assuming that the original image is $y$, the MP algorithm is used to decompose the image. The decomposition process is as follows. Firstly, the atom most related to the original image $y$ is found from the over complete dictionary, that is, the atom with the largest inner product of the image $y$. Then, the atoms are selected and the reconstruction residuals are obtained. Therefore, the image $y$ is decomposed into two parts.

$$
\begin{gathered}
\left|<y, d_{1}>\right|=\sup \left|<y, d_{r}>\right| \\
y=<y, d_{1}>d_{1}+R_{1} y
\end{gathered}
$$

Formula(5) represents the atom selected from the atomic library with the largest inner product of the original image $y, d_{i}$ represents any atom in the atomic library, and $d_{1}$ represents the atom most related to $y$ selected in the first iteration. Formula (6) represents the decomposition of the original image $y$, $<y, d_{1}>d_{1}$ represents the projection component of the original image $y$ on the selected atom $d_{1}$, and $R_{1} y$ represents the residual value. It can be seen from equation (6) that the best atom $d_{1}$ and residual $R_{1} y$ are orthogonal.

$$
\|y\|_{2}^{2}=\left|<y, d_{1}>\right|^{2}+\left\|R_{1} y\right\|_{2}^{2}
$$

It is because of the orthogonality of $d_{1}$ and $R_{1} y$ that the iteration can continue and the best atom selected in the next iteration is no longer $d_{1}$, which avoids the iteration into a dead cycle. Next, the residual $R_{1} y$ is iterated according to the above method, and the atom $d_{2}$ most related to $R_{1} y$ is selected from the over complete dictionary.

$$
R_{1} y=<R_{1} y, d_{2}>d_{2}+R_{2} y
$$

Similarly, $d_{2}$ and $R_{2} y$ satisfy orthogonality,

$$
\left\|R_{1} y\right\|_{2}^{2}=\left|<R_{1} y, d_{2}>\right|^{2}+\left\|R_{2} y\right\|_{2}^{2}
$$

The same method is used to iterate the residuals for many times until the residuals reach the required value or 0. Finally, the original image can be decomposed into a linear combination of the selected atoms.

$$
y=\sum_{n=0}^{k}<R_{n} y, d_{n}>d_{n}+R_{n} y
$$

In the above iterative process, the atoms selected in each iteration are most related to the residual obtained in the last iteration. Therefore, the combination of atoms selected in the iteration can be used to represent the original image linearly, so as to reconstruct the original image.

MP algorithm is simple in principle and easy to implement. Compared with other algorithms, its fast speed is one of its advantages. However, MP algorithm in the face of some more complex images, the reconstruction effect is not very good, and its peak signal-to-noise ratio also decreases with the decrease of sampling rate. The main reason for the unsatisfactory 
reconstruction effect of MP algorithm is that it can not guarantee that the atoms selected from the atomic library are orthogonal to each other, so it can not guarantee that each iteration result is optimal. Therefore, in order to obtain better reconstruction effect, more iterations and higher sampling rate are needed.

(3) Orthogonal Matching Pursuit.

Orthogonal matching tracking is an improved algorithm for matching tracking. The algorithm is the same as matching tracking algorithm in atom selection strategy. The residual update in matching tracking algorithm is improved. The difference between the two algorithms is that the orthogonal matching tracking algorithm can ensure that the selected atoms are orthogonal to each other. The algorithm will orthogonally process all the selected atoms before projecting the signal to ensure that the signal is projected on the orthogonal atomic space, so as to ensure that the residual is orthogonal to all the atoms selected before, while the matching tracking algorithm can only ensure that the residual is orthogonal to each other At present, the orthogonality of selected atoms cannot be guaranteed.

OMP algorithm is also an iterative algorithm for signal reconstruction. In each iteration process, the best atom selected is the one with the largest inner product value with the current residual, and then the relevant part is subtracted from the observed value to update the residual until the number of iterations reaches the sparsity $\mathrm{K}$, and then the iteration is stopped. Because the algorithm will orthogonalize all the searched atoms in the residual updating process, which greatly improves the complexity of OMP algorithm, resulting in longer signal reconstruction time.

The specific iteration process of OMP algorithm is as follows.

STEP1. Initialize residuals $r_{0}=y$. Index set is $\Lambda_{0}=\phi$,

iteration count $t=1$, matching matrix $D_{0}=\Phi$.

STEP2. Find the index $\lambda_{t}$ that satisfies the optimization problem, $\lambda_{t}=\arg \max _{j=1 . . n}\left|<r_{t-1}, \Phi_{j}>\right|$.

STEP3. Update index set and matching matrix, $\Lambda_{t}=\Lambda_{t-1} \cup\left\{\lambda_{t}\right\}$ and $D_{t}=\left[D_{t-1}, \Phi_{\lambda_{t}}\right]$

STEP4. Solve the least square problem, $x_{t}=\arg \min \left\|y-D_{t} x_{t}\right\|_{2}$

STEP5. Calculate update residuals, $r_{t}=y-D_{t} x_{t}$

STEP6. Update iterations $t=t+1$. Judge whether the iteration stop condition is satisfied. If the condition satisfied, output $\hat{x}=x_{t}$, otherwise return to Step 2 .

(4) Piecewise Orthogonal Matching Tracking.

OMP algorithm improves MP algorithm to a great extent. However, due to the orthogonality of all selected atoms in each iteration, the computation is very heavy in the face of largescale problems, which leads to the slow process of sparse decomposition and is difficult to be applied in practice. OMP algorithm is improved in the residual update phase of MP algorithm, but its atom selection strategy is still the same as MP algorithm, and only one atom with the highest matching degree is selected from the atom Library in each iteration. Although this selection method can ensure the reconstruction effect of signal, its cost is that it takes a long time.

In view of the shortcomings of OMP algorithm, the stomp algorithm proposed by D. Donoho et al. Greatly simplifies the OMP algorithm. Piecewise orthogonal matching pursuit (stomp) is an improvement of orthogonal matching pursuit (OMP) algorithm, which further reduces the complexity of the algorithm and provides a new method for solving large-scale sparse decomposition problems. In the piecewise orthogonal matching pursuit algorithm, instead of iterating all the time, the number of iterations is limited. In the process of each iteration, not only one atom is selected for iteration, but a threshold is set to control the selection of atoms. The selected atoms are all the atoms whose matching degree is higher than the threshold, and the atoms whose matching degree is lower than the threshold will be discarded It greatly improves the running speed.

The core steps of the algorithm are as follows.

STEP1.Initialize the parameters, reconstructed signal $\hat{X}=0$, Initialization residuals $r_{0}=y$, Index set $\Lambda_{0}=\phi$, iteration count $t=1$.

STEP2. Find the index. The index of all atoms whose matching degree is greater than the threshold $J_{t}=\left\{\lambda:\left|<r_{t-1}, \varphi_{j}>\right|>\varsigma\right\}, J_{t}$ is the index set selected.

STEP3.Update index set $\Lambda_{t}=\Lambda_{t-1} \cup\left\{J_{t}\right\}$.

STEP4.Update residuals $r_{t}=y-\Phi_{\Lambda_{t}}\left(\Phi_{\Lambda_{t}}^{+} y\right)$.

STEP5.Check the iteration stop condition. If the condition satisfied, stop the iteration and output $\hat{X}=\Phi_{\Lambda_{t}}^{+} y$, otherwise return to Step 2.

\section{Sparse Image Reconstruction Algorithm}

In the last section, several commonly used image reconstruction algorithms are introduced in detail. It can be seen that the prior information of these algorithms is that the sparsity of the image is known. However, in practice, the sparsity of the image is often unknown or difficult to obtain accurately. For the problem of image reconstruction with unknown sparsity, this section proposes a sparse image reconstruction algorithm.

Greedy tracking algorithm has been paid close attention. MP algorithm is the first greedy algorithm, and OMP algorithm is improved. The speed of image reconstruction has been greatly improved, which greatly saves time, but the reconstruction effect is not very ideal. The improvement of the algorithm further reduces the complexity of the reconstruction algorithm, and lays the foundation for the application in large-scale signal processing. These greedy algorithms are only applicable when the prior information sparsity of the signal is known. However, in practical problems, the sparsity of the signal is often unknown, which greatly reduces the value of the existing greedy reconstruction methods in signal reconstruction.

In view of the situation that the sparsity of greedy algorithm is unknown, a sparse reconstruction algorithm is proposed, which introduces the idea of sparsity theory into the piecewise orthogonal matching tracking algorithm to realize the sparsity 
estimation and image reconstruction, so that the image signal with unknown sparsity can be reconstructed quickly and accurately. The residual is initialized, the input image is matched and filtered according to the set threshold, and then the candidate set is established by using the obtained atoms. On this basis, the step size is updated according to the residual attenuation judgment to obtain the support set, and finally the image is reconstructed according to the new support set. The structure of the algorithm is shown in Figure 2.

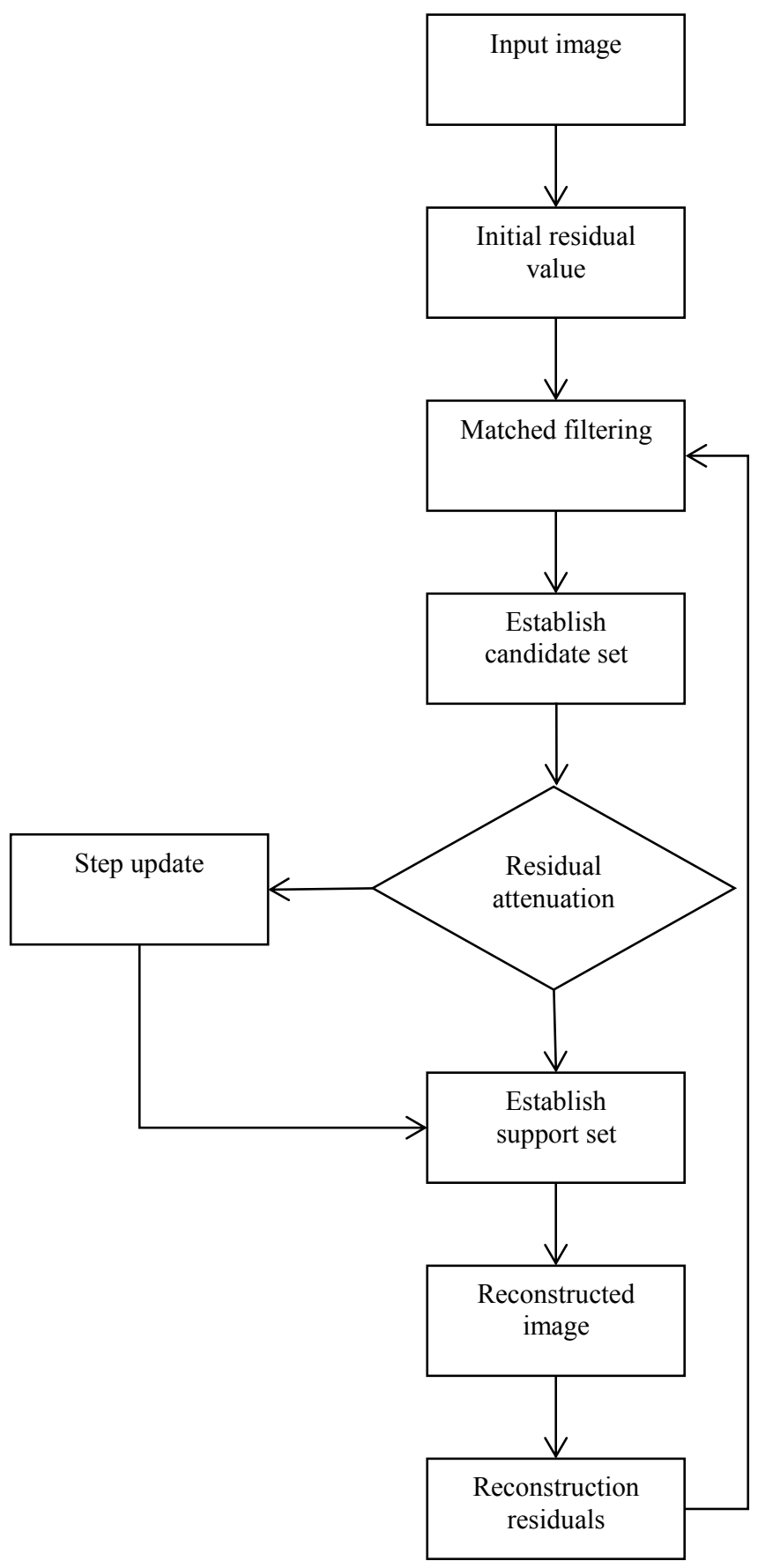

Figure 2. Structure of sparse reconstruction algorithm

Assuming that the input image is $y$, initialization residuals $r_{0}=y, D$ is a complete dictionary, support set $F=\phi$, and the threshold is set according to the residuals to perform matched filtering.

$$
J_{k}=\left\{\mathrm{j}:\left|\Phi^{T} \bullet r_{k-1}\right|>\mathrm{t}_{k} \sigma_{k}\right\}
$$

After matching filtering, $D_{J_{k}}$ and the sum of atoms whose matching degree is higher than the threshold $t_{k} \sigma_{k}$ are selected, and then the candidate set $C_{k}$ is established according to the selected matching atoms.

$$
C_{k}=F_{k-1} \cup D_{J_{k}}
$$

Among them, $C_{k}$ is the candidate set, $F_{k-1}$ is the support set, and $D_{J_{k}}$ is the atom set obtained by the matched filter.

After the candidate set is obtained, the support set is updated according to whether the residual is decaying or not. If the residual is decaying, the number of candidate sets is directly used.

$$
F_{k}=\operatorname{Max}\left(\left|D_{C_{k}}^{T} \bullet y\right|, S_{k}\right)
$$

Among them, $S_{k}$ represents the number of atoms, and support sets $F_{k}$ represent the set of atoms selected and matched for step size $S_{k}$ in the dictionary of atomic composition $y$ in candidate set.

When the atoms in the support set are orthogonalized, the image is approximated by the approximation method, so as to realize the image reconstruction and obtain the reconstruction residual.

$$
\begin{gathered}
x_{k}=D_{F_{k}}^{+} y \\
r_{k}=y-\Phi_{F_{k}}^{T} x_{k}
\end{gathered}
$$

Finally, the iteration termination condition is judged, that is $\|r\|<\varepsilon$, and $\varepsilon$ is a given value.

The algorithm steps can be expressed.

STEP1. The initialization parameter takes the input image as the initial residual.

STEP2. Matching filtering to obtain the atom most related to the residual.

STEP3. Using the atoms obtained in STEP 2 to establish a candidate set.

STEP4. Judge whether the residual is attenuated or not.

STEP5. If the judgment in STEP 4 is yes, the support set is established by using the candidate set obtained in STEP 3, and the process goes to STEP 8 .

STEP6. If the STEP 4 is judged as no, the step size is updated. STEP7. Establish the support set, and enter STEP 8.

STEP8. The image is approximated by the support set to get the reconstructed image.

STEP9. Using the reconstructed image obtained in STEP 8 to calculate the reconstruction residual.

STEP10. Return to STEP 2, take the reconstruction residuals obtained in STEP 9 as the residuals to iterate until the two 
reconstruction residuals are less than the given value, and get the best reconstructed image.

\section{EXPERIMENT}

Two gray images of Peppers and Baboon are selected as test images in this paper, and the reconstruction effect and performance of the improved algorithm is tested by peak signalto-noise ratio (PSNR) and reconstruction time (t). The algorithm is programmed with MATLAB.

The following is the effect using the proposed algorithm on Peppers and Baboon images.

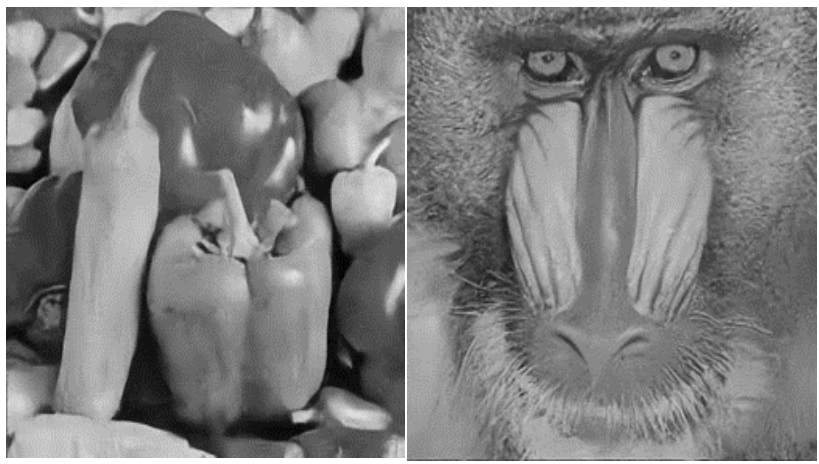

(a) Original image

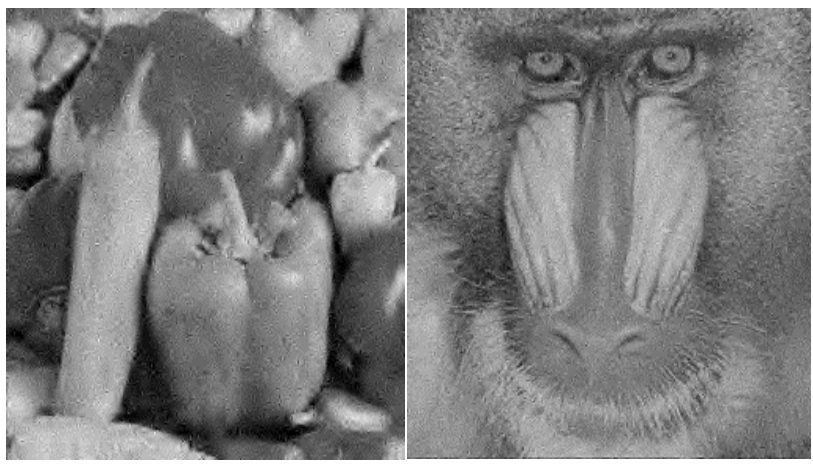

(b) Image at sampling rate of 0.5
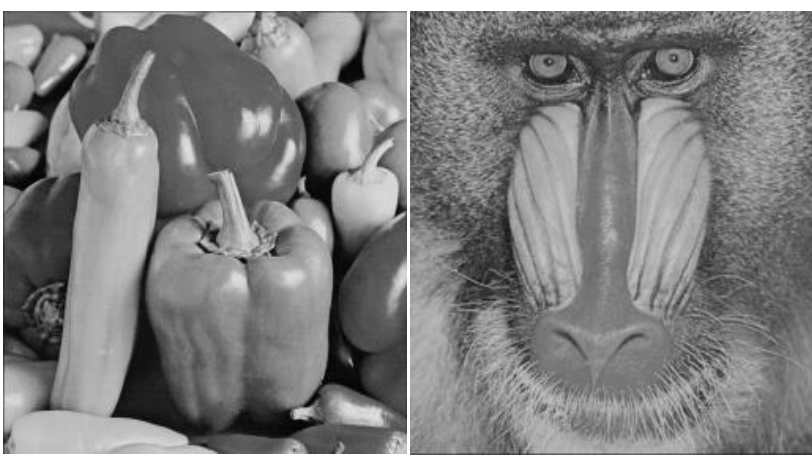

(c) Reconstruction image of improved algorithm

Figure 3. Comparison of image reconstruction effect of improved algorithm

The above results are only experiments on the improved algorithm. For obtaining the exhibition of the reconstruction effect, the improved algorithm is compared with $\mathrm{BP}, \mathrm{OMP}$ and
StOPM algorithm. The sampling rate is 0.5 during the experiment, and the experimental results of the improved algorithm on Ship images are shown in Figure 4.

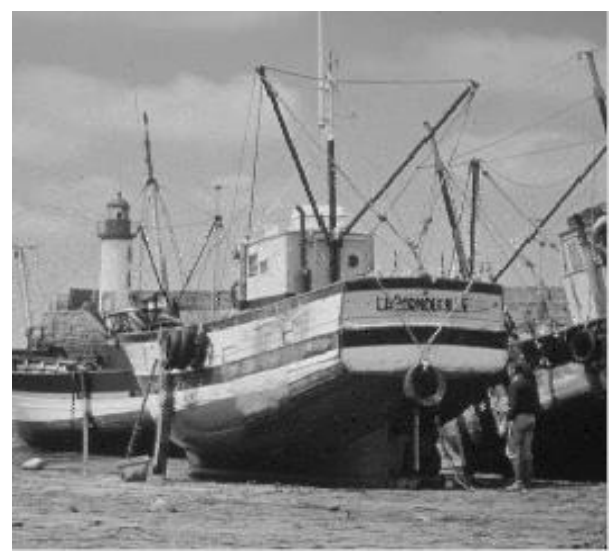

(a) Original image

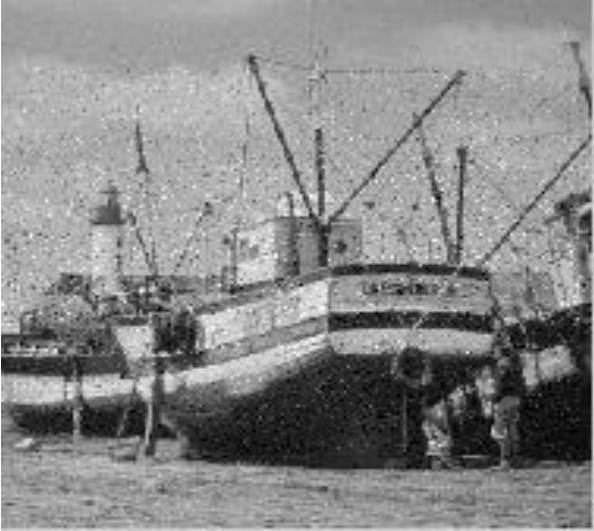

(b) Reconstruction effect of BP algorithm

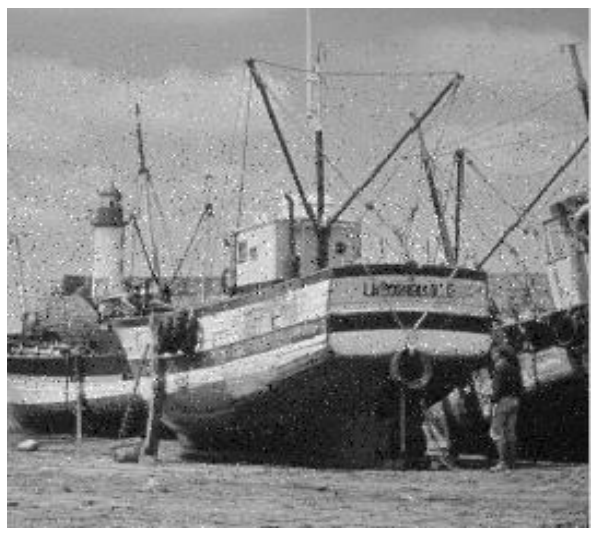

(c) Reconstruction effect of OMP algorithm 


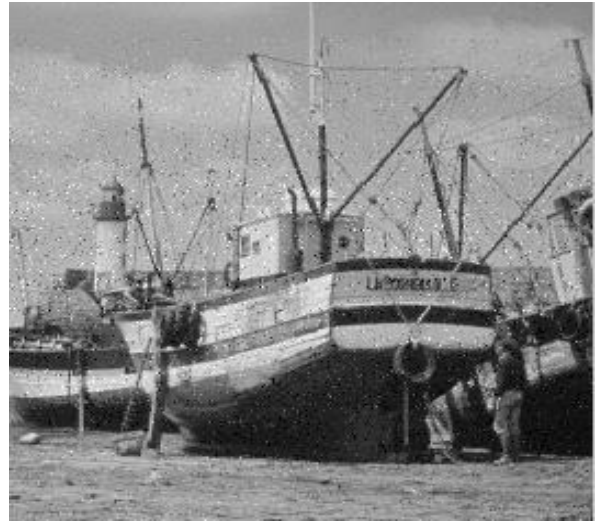

(d) Reconstruction effect of StOPM algorithm

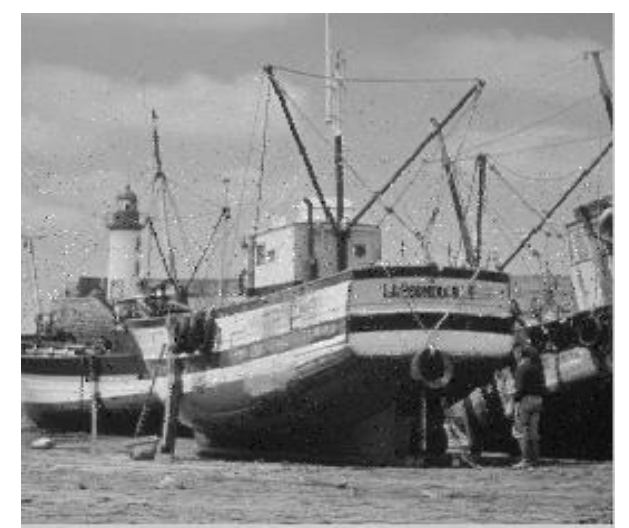

(e) Reconstruction effect of improved algorithm

Figure 4. The reconstruction effect of ship

The above figure illustrates the effect of reconstruction under different algorithms on the two gray images of aircraft and shift at the sampling rate of 0.5 . The image reconstruction efficiency of this algorithm under different levels of noise is better than other algorithms. In addition, the details of the reconstructed image are clearer, and this advantage is more significant when the noise increases. The standard deviations of Gaussian noise are $0,2,4$ and 6 , respectively. The regularization parameters in the corresponding sparse representation model are changed accordingly, and the values are $0.2,0.2,0.4$ and 0.6 respectively; According to the data results, in the process of Gaussian noise gradually increasing, the MSE values of the three algorithms increase, while the PSNR and SSIM values decrease, and they are all in the direction of degradation. But through the comparison, it can be found that in general, the reconstruction effect of the algorithm in this paper is dominant, only when the noise is abnormally large, the quality is relatively fast. Under different levels of noise, the anti Gaussian noise ability of the algorithm is enhanced. Among them, the best reconstruction effect is the improved algorithm.

This paper gives the peak signal-to-noise ratio (PSNR) value and reconstruction time $(\mathrm{T})$ of two images at the sampling rate of 0.5 , as shown in Table 1 and Table 2 .

Table 1. PSNR ( $\mathrm{db}$ ) values of different algorithms at the same sampling rate

\begin{tabular}{|c|c|c|c|c|}
\hline Images & \multicolumn{4}{|c|}{ Different algorithms } \\
\cline { 2 - 5 } & BP & OMP & StOPM & $\begin{array}{l}\text { Improved } \\
\text { algorithm }\end{array}$ \\
\hline Ship & 20.055 & 22.376 & 24.506 & 27.361 \\
\hline
\end{tabular}

Table 2. Reconstruction time $t$ (s) of different algorithms at the same sampling rate

\begin{tabular}{|c|c|c|c|c|}
\hline \multirow{2}{*}{ Images } & \multicolumn{4}{|c|}{ Different algorithms } \\
\cline { 2 - 5 } & BP & OMP & StOPM & $\begin{array}{l}\text { Improved } \\
\text { algorithm }\end{array}$ \\
\hline Ship & 35.893 & 11.256 & 3.027 & 0.982 \\
\hline
\end{tabular}

It can be seen more vividly from the chart that the PSNR value and reconstruction time $t$ of different algorithms at the same sampling rate. Because of introducing the advantage of sparse representation in the iterative process, the improved algorithm can not only reconstruct the image with unknown sparsity, but also has advantages over other algorithms in reconstruction time. The reconstruction time of the improved algorithm is the shortest under the same sampling rate, and the reconstruction effect is better than that of other algorithms.

\section{CONCLUSION}

This paper mainly proposes an image reconstruction algorithm based on sparse representation. Firstly, the principle of image reconstruction algorithm based on sparse representation is introduced, and then the current commonly used reconstruction algorithms BP, MP, OMP and StOPM are described in detail. Finally, a reconstruction algorithm based on sparse representation is proposed. By introducing the sparsity theory and dynamically changing the step size to approximate the sparsity, the algorithm can still process the image when the sparsity is unknown. The reconstruction time of the improved algorithm is the shortest under the same sampling rate, and the reconstruction effect is better than that of other algorithms.

The existing image is based on the known image observation model, image acquisition and error estimation of the final image are based on the degradation model. It is assumed that the image degradation is all through blur and down sampling, and the noise introduced is generally Gaussian noise. This model is too idealized to reflect the special situation in practical application. Therefore, it is necessary to further study the prior knowledge of image, and accurately estimate the noise interference, motion parameters and sensor imaging process that may occur in the actual degradation process.

\section{REFERENCES}

[1] He N A, Wang R B, Wang Y C. "Dynamic MRI reconstruction exploiting blind compressed sensing combined transform learning regularization ScienceDirect", Neurocomputing, 2020, 392, pp. 160-167.

[2] Peper E S, Gottwald L M, Zhang Q, et al. "Highly accelerated 4D flow cardiovascular magnetic resonance using a pseudo-spiral Cartesian acquisition and compressed sensing reconstruction for carotid flow and wall shear stress", Journal of Cardiovascular Magnetic Resonance, 2020, 22, pp. 2278-2324. 
[3] Kavitha T S , Prasad K S. "Hybridizing ant lion with whale optimization algorithm for compressed sensing MR image reconstruction via 11 minimization: an ALWOA strategy", Evolutionary Intelligence, 2020, 7, pp.1-11.

[4] Onodera M , K Aratani, Shonai T , et al. "Lateral Position With Gantry Tilt Further Improves Computed Tomography Image Quality Reconstructed Using Single-Energy Metal Artifact Reduction Algorithm in the Oral Cavity", Journal of Computer Assisted Tomography, 2020, 44, pp. 553-558.

[5] N Ilakiyaselvan, AN Khan, A Shahina, "Deep learning approach to detect seizure using reconstructed phase space images", Journal of Biomedical Research, 2020, 34, pp. 238-249.

[6] Narita A , Ohkubo M . "A pitfall of using the circular- edge technique with image averaging for spatial resolution measurement in iteratively reconstructed CT images", Journal of Applied Clinical Medical Physics, 2020, 21, pp. 77-90

[7] Allemang A, Thacker R, Demarco R A, et al. "The 3D reconstructed skin micronucleus assay using imaging flow cytometry and deep learning: a proof-of-principle investigation", Mutation Research/Genetic Toxicology and Environmental Mutagenesis, 2021:503314.

[8] Dhont, Verellen D, Mollaert I, et al. "RealDRR - Rendering of realistic digitally reconstructed radiographs using locally trained image-to-image translation", Radiotherapy and Oncology, 2020, 153, pp. 352-360.

[9] Zhao, Huang, Liu, et al. "A sparse representation-based fusion model for improving daily MODIS C6.1 aerosol products on a $3 \mathrm{~km}$ grid", International Journal of Remote Sensing, 2021 285, pp. 204-219.

[10] Yang J , Tse P . "Sparse representation of complex steerable pyramid for machine fault diagnosis by using non-contact video motion to replace conventional accelerometers", Measurement, 2021, 365:109104.

[11] Zheng S, Zhang Y, Liu W, et al. A Dictionary Learning Algorithm Based on Dictionary Reconstruction and Its Application in Face Recognition. Mathematical Problems in Engineering, 2020, 2020, pp.1-13.

[12] Wang G , Han H, Carranza E, et al. Tensor-Based Low-Rank and Sparse Prior Information Constraints for Hyperspectral Image Denoising. IEEE Access, 2020, PP(99):1-1

[13] Li B, Rencker L, Dong J , et al. Sparse Analysis Model Based Dictionary Learning for Signal Declipping. IEEE Journal of Selected Topics in Signal Processing, 2021, PP(99):1-1.

[14] Maqsood S , Javed U . Multi-modal Medical Image Fusion based on Twoscale Image Decomposition and Sparse Representation. Biomedical Signal Processing and Control, 2020, 57:101810.

[15] Huynh T , Saab R . Fast binary embeddings, and quantized compressed sensing with structured matrices. Communications on Pure and Applied Mathematics, 2020, 73, pp. 231-244.

[16] Yu H, Gao L, Liao W, et al. Global Spatial and Local Spectral SimilarityBased Manifold Learning Group Sparse Representation for Hyperspectral Imagery Classification. IEEE Transactions on Geoscience and Remote Sensing, 2020, 121, 854-863.

[17] Rafeek Mamdouh, Hazem M. El-Bakry, Alaa Riad, Nashaat El-Khamisy, Converting 2D-Medical Image Files "DICOM" into 3D- Models, based on Image Processing, and Analysing their Results with Python Programming, WSEAS Transactions on Computers, Volume 19, 2020, Art. \#2, pp. 1020.

\section{Creative Commons Attribution License 4.0 (Attribution 4.0 International, CC BY 4.0)}

This article is published under the terms of the Creative Commons Attribution License 4.0

https://creativecommons.org/licenses/by/4.0/deed.en_US 